8-3-2020

\title{
A New Two Parametric Weighted Generalized Entropy for Lifetime Distributions
}

Bilal Ahmad Bhat

University of Kashmir, Srinagar, India, bilal3819md@gmail.com

Mirza Abdul Khaliq Baig

University of Kashmir, Srinagar, India, baigmak@gmail.com

Follow this and additional works at: https://digitalcommons.wayne.edu/jmasm

Part of the Applied Statistics Commons, Social and Behavioral Sciences Commons, and the Statistical Theory Commons

\section{Recommended Citation}

Bhat, B. A., \& Baig, M. A. K. (2019). A new two parametric weighted generalized entropy for lifetime distributions. Journal of Modern Applied Statistical Methods, 18(2), eP2757. doi: 10.22237/jmasm/ 1604189340

This Regular Article is brought to you for free and open access by the Open Access Journals at DigitalCommons@WayneState. It has been accepted for inclusion in Journal of Modern Applied Statistical Methods by an authorized editor of DigitalCommons@WayneState. 


\section{A New Two Parametric Weighted Generalized Entropy for Lifetime Distributions}

\author{
Bilal Ahmad Bhat \\ University of Kashmir \\ Srinagar, India
}

\author{
Mirza Abdul Khaliq Baig \\ University of Kashmir \\ Srinagar, India
}

The concept of weighted generalized entropy and its dynamic residual (version) is developed. The general expressions of these two uncertainty measures corresponding to some well-known lifetime distributions are derived. It is shown that the proposed dynamic entropy determines the survival function uniquely. Some significant properties and inequalities of this dynamic entropy are also discussed.

Keywords: Shannon's entropy, lifetime distributions, residual entropy, length-biased entropy, characterization results

\section{Introduction}

The notion of entropy introduced by Shannon (1948) has an important role in the context of information theory and also in other sciences. If $X$ is an absolutely continuous non-negative r.v. having p.d.f. $\mathrm{f}(x)$, then the Shannon's entropy is defined as

$$
\mathrm{H}(X)=-\int_{0}^{\infty} \mathrm{f}(x) \log \mathrm{f}(x) d x=-\mathrm{E}[\log \mathrm{f}(X)]
$$

Throughout this article, the r.v. $X$ and the p.d.f refer to absolutely continuous nonnegative random variable and probability density function, respectively.

In information theory, various generalizations of (1) were proposed and, consequently, in this article a new two parametric generalization of this uncertainty measure is developed as follows:

doi: 10.22237/jmasm/1604189340 | Accepted: February 17, 2018; Published: August 3, 2020.

Correspondence: Bilal Ahmad Bhat, bilal3819md@gmail.com 


\section{BHAT \& BAIG}

$$
\mathrm{H}_{(\alpha, \beta)}(X)=\frac{\alpha}{\beta(\beta-\alpha)} \log \int_{0}^{\infty} \mathrm{f}^{\frac{\alpha}{\beta}}(x) d x, \quad 0<\alpha<\beta, \beta \geq 1,
$$

where

$$
\lim _{\substack{\alpha \rightarrow 1 \\ \beta=1}} \mathrm{H}_{(\alpha, \beta)}(X)=-\int_{0}^{\infty} \mathrm{f}(x) \log \mathrm{f}(x) d x
$$

is the well-known basic entropy given in (1).

As argued by Ebrahimi (1996), if a system with the lifetime $X$ is surviving at time $t$, then the measure (1) is not suitable for ascertaining the uncertainty about the remaining life $X_{t}=[X-t \mid X>t]$ of such a system. Therefore, the concept of residual entropy was introduced on the residual lifetime $X_{t}=[X-t \mid X>t]$, which is given by

$$
\mathrm{H}(X ; t)=-\int_{t}^{\infty} \frac{\mathrm{f}(x)}{\overline{\mathrm{F}}(t)} \log \frac{\mathrm{f}(x)}{\overline{\mathrm{F}}(t)} d x
$$

where, $\overline{\mathrm{F}}(t)=1-\mathrm{F}(t)$ represents the survival function (s.f.) of $X$. In the same way and on the basis of (2), the generalized entropy for the residual lifetime $X_{t}=[X-t \mid X>t]$ is defined as

$$
\mathrm{H}_{(\alpha, \beta)}(X ; t)=\frac{\alpha}{\beta(\beta-\alpha)} \log \left(\int_{t}^{\infty}\left(\frac{\mathrm{f}(x)}{\overline{\mathrm{F}}(t)}\right)^{\frac{\alpha}{\beta}} d x\right), \quad 0<\alpha<\beta, \beta \geq 1
$$

Shannon's entropy provides the equal importance or weight to the occurrence of every event of a probabilistic experiment with respect to the goal of the experimenter. Sometimes it is essential to also consider the importance of the elementary events. Therefore, an alternative measure, known as weighted or lengthbiased entropy (WE) which considers both the objective probabilities and the real qualitative weights of the elementary events was introduced by Belis and Guiasu (1968) as 


$$
\begin{aligned}
\mathrm{H}^{\mathrm{w}}(X) & =-\int_{0}^{\infty} \mathrm{w}(x) \mathrm{f}(x) \log \mathrm{f}(x) d x \\
& =-\int_{0}^{\infty} x \mathrm{f}(x) \log \mathrm{f}(x) d x=-\mathrm{E}[X \log \mathrm{f}(X)]
\end{aligned}
$$

where the coefficient $x$ in the integrand, which is actually the length of the observed r.v. $X$, represents the weight function $\mathrm{w}(x)$ or importance of the occurrence of the event $X=x$ and hence introduces the existence of length-biased or weighted entropy.

The weighted residual entropy or the length-biased version of (3) by Di Crescenzo and Longobardi (2006) is given by

$$
\mathrm{H}^{\mathrm{w}}(X ; t)=-\int_{t}^{\infty} x \frac{\mathrm{f}(x)}{\overline{\mathrm{F}}(t)} \log \frac{\mathrm{f}(x)}{\overline{\mathrm{F}}(t)} d x .
$$

A variety of length-biased or weighted uncertainty measures have been developed and applied to measuring the uncertainty corresponding to different type of probability distributions. For more details see Mirali and Baratpour (2017), Misagh et al. (2011), Kayal (2018), Misagh and Yari (2011), Nair et al. (2017), Nourbakhsh and Yari (2016), Shiwei and Ting-Zhu (2017), Khammar and Jahanshahi (2018) and Yasaei Sekeh et al. (2012). Therefore, the objective of this study is to develop a new two parametric weighted generalized entropy and its dynamic version.

\section{Weighted Generalized Entropy}

Consider the weighted version of GE (2) which leads to the weighted generalized entropy (WGE). The general expressions of this WGE corresponding to some particular lifetime distributions are now considered. Analogous to WE (5) and on the basis of (2), the WGE is given by

$$
\mathrm{H}_{(\alpha, \beta)}^{\mathrm{w}}(X)=\frac{\alpha}{\beta(\beta-\alpha)} \log \left(\int_{0}^{\infty}(x \mathrm{f}(x))^{\frac{\alpha}{\beta}} d x\right), \quad 0<\alpha<\beta, \beta \geq 1
$$

where the coefficient $x$ is defined in (5). 


\section{BHAT \& BAIG}

Remark 1. A new way of expressing $\mathrm{H}_{(\alpha, \beta)}^{\mathrm{w}}(X)$ is as follows:

$$
\begin{aligned}
\mathrm{H}_{(\alpha, \beta)}^{\mathrm{w}}(X) & =\frac{a}{\beta(\beta-\alpha)} \log \int_{0}^{\infty}(x \mathrm{f}(x))^{\frac{\alpha}{\beta}} d x \\
& =\frac{\alpha}{\beta(\beta-\alpha)} \log \mathrm{E}\left(X^{\frac{\alpha}{\beta}} \mathrm{f}^{\frac{a}{\beta}-1}(X)\right)
\end{aligned}
$$

The following example exhibits the comparison of GE (2) with its weighted version (7).

Example 1. Let the two r.v.s $X$ and $Y$ be distributed as follows:

$$
\mathrm{f}_{X}(x)=\left\{\begin{array}{ll}
\frac{1}{2} x, & 0<x<2 \\
0, & \text { otherwise }
\end{array} \text { and } \mathrm{f}_{Y}(y)= \begin{cases}\frac{1}{2}(2-y), & 0<y<2 \\
0, & \text { otherwise }\end{cases}\right.
$$

Table 1. Comparison between GE and WGE

\begin{tabular}{rrrrrr}
$\boldsymbol{\alpha}$ & $\boldsymbol{\beta}$ & $\mathbf{H}_{(\alpha, \beta)}(\boldsymbol{X})$ & $\mathbf{H}_{(\alpha, \beta)}(\boldsymbol{\eta})$ & $\mathbf{H}_{(\alpha, \beta)}^{\mathrm{w}}(\boldsymbol{X})$ & $\mathbf{H}_{(\alpha, \beta)}^{\mathrm{w}}(\boldsymbol{Y})$ \\
\hline 0.4 & 1.2 & 0.169 & 0.169 & 0.172 & 0.121 \\
& 1.4 & 0.126 & 0.126 & 0.125 & 0.098 \\
& 1.6 & 0.098 & 0.098 & 0.096 & 0.080 \\
& 1.8 & 0.078 & 0.078 & 0.761 & 0.066 \\
& 2.0 & 0.064 & 0.064 & 0.062 & 0.056 \\
0.6 & 1.3 & 0.207 & 0.207 & 0.237 & 0.097 \\
& 1.5 & 0.159 & 0.159 & 0.170 & 0.095 \\
& 1.7 & 0.125 & 0.125 & 0.130 & 0.086 \\
& 1.9 & 0.102 & 0.102 & 0.103 & 0.075 \\
& 2.1 & 0.084 & 0.084 & 0.084 & 0.065 \\
& & & & & \\
& 1.6 & 0.180 & 0.180 & 0.217 & 0.066 \\
& 1.8 & 0.145 & 0.145 & 0.162 & 0.074 \\
& 2.0 & 0.119 & 0.119 & 0.128 & 0.071 \\
& 2.2 & 0.099 & 0.099 & 0.104 & 0.066 \\
& 2.4 & 0.084 & 0.084 & 0.086 & 0.060 \\
\hline
\end{tabular}


TWO PARAMETRIC ENTROPY FOR LIFETIME DISTRIBUTIONS

Table 1 (continued).

\begin{tabular}{rrrrrr}
$\boldsymbol{\alpha}$ & $\boldsymbol{\beta}$ & $\mathbf{H}_{(\alpha, \beta)}(\boldsymbol{X})$ & $\mathbf{H}_{(\alpha, \beta)}(\boldsymbol{\eta})$ & $\mathbf{H}_{(\alpha, \beta)}^{\mathrm{w}}(\boldsymbol{X})$ & $\mathbf{H}_{(\alpha, \beta)}^{\mathrm{w}}(\boldsymbol{Y})$ \\
\hline 1.2 & 2.5 & 0.111 & 0.111 & 0.130 & 0.047 \\
& 2.6 & 0.145 & 0.145 & 0.118 & 0.048 \\
& 2.7 & 0.119 & 0.119 & 0.108 & 0.049 \\
& 2.8 & 0.099 & 0.099 & 0.099 & 0.049 \\
& 2.9 & 0.084 & 0.084 & 0.092 & 0.048 \\
& & & & & \\
& 3.0 & 0.090 & 0.090 & 0.104 & 0.041 \\
& 3.2 & 0.080 & 0.080 & 0.089 & 0.042 \\
& 3.4 & 0.072 & 0.072 & 0.078 & 0.041 \\
& 3.6 & 0.064 & 0.064 & 0.068 & 0.040 \\
& 3.8 & 0.058 & 0.058 & 0.061 & 0.038 \\
& 3.2 & 0.090 & 0.090 & 0.108 & 0.033 \\
& 3.4 & 0.080 & 0.080 & 0.093 & 0.036 \\
& 3.6 & 0.072 & 0.072 & 0.081 & 0.037 \\
& 3.8 & 0.065 & 0.065 & 0.072 & 0.037 \\
& 4.0 & 0.059 & 0.059 & 0.064 & 0.036 \\
\hline
\end{tabular}

In Table 1, corresponding to the above distributions, we examine that for different possible values of the parameters $\alpha$ and $\beta$, even though the GE of the r.v. $X$ is same as that of $Y$, but their WGEs are not identical.

Proposition 1. For $m>0$, the following equality holds:

$$
\mathrm{H}_{(\alpha, \beta)}^{\mathrm{w}}(m X)=\frac{\alpha}{\beta(\beta-\alpha)} \log m+\mathrm{H}_{(\alpha, \beta)}^{\mathrm{w}}(X) .
$$

In Table 2, consider the general expressions of WGE for some particular probability distributions. Also, note that

$$
r=\frac{\alpha}{\beta(\beta-\alpha)}, \quad m=\frac{\alpha}{\beta}, \quad \text { and } \quad \Gamma(b, a z)=a^{b} \int_{z}^{\infty} \mathrm{e}^{-a x} x^{b-1} d x, a, b>0
$$

is an upper incomplete gamma function. 


\section{BHAT \& BAIG}

Table 2. The expressions of WGE for some lifetime distributions

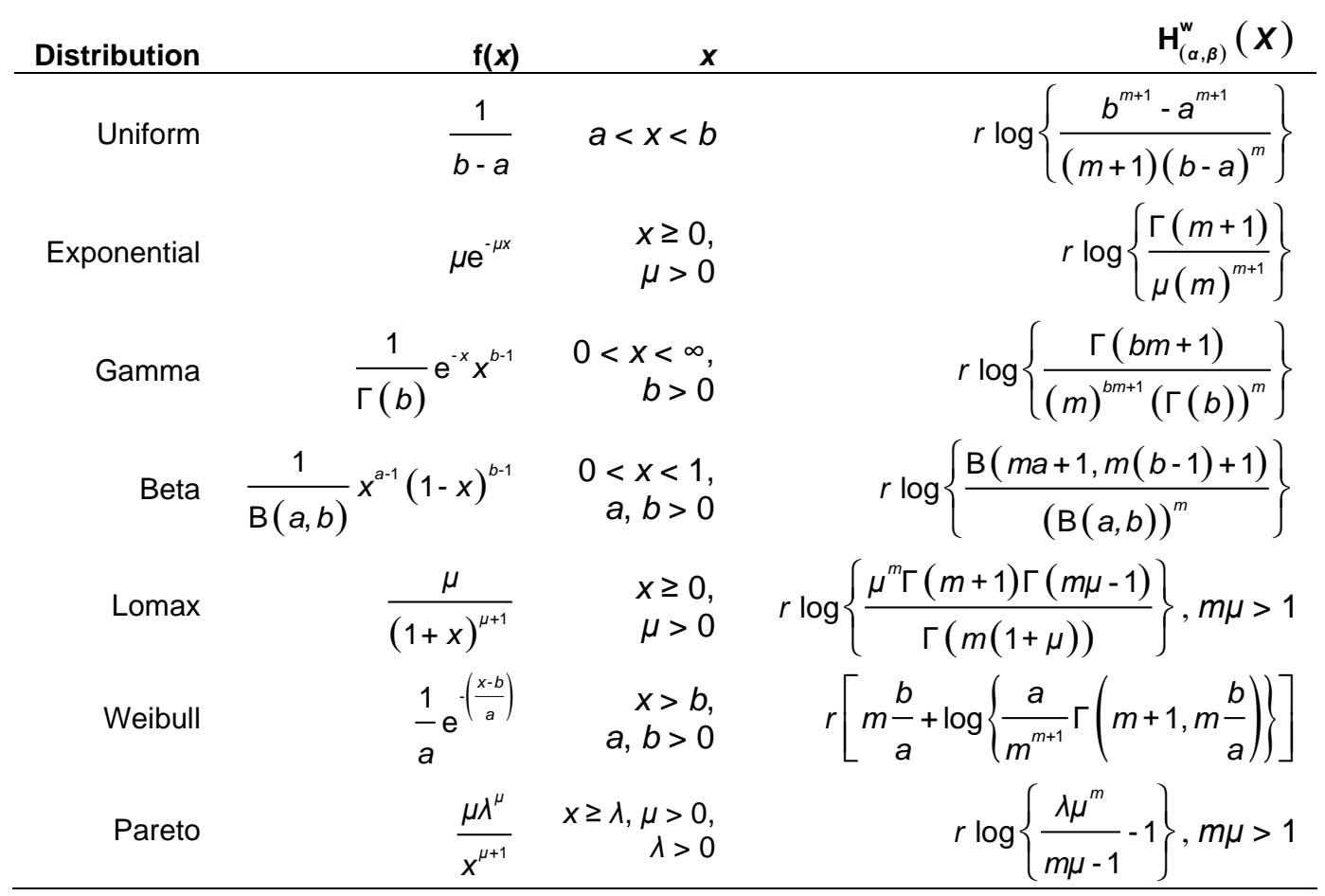

Note: $\mathrm{B}(a, b)=\int_{0}^{1} x^{a-1}(1-x)^{b-1} d x=\frac{\Gamma(a) \Gamma(b)}{\Gamma(a+b)} a, b>0$ and $\Gamma(a)$ is the gamma function

\section{Weighted Generalized Residual Entropy (WGRE)}

Consider the dynamic (residual) version of WGE (7) and also focus on a characterization result which shows that this dynamic measure determines the survival function uniquely.

Definition 1. Analogous to (6), the dynamic (residual) version of (7) is given by

$$
\mathrm{H}_{(\alpha, \beta)}^{\mathrm{w}}(X ; t)=\frac{\alpha}{\beta(\beta-\alpha)} \log \left(\int_{t}^{\infty}\left(x \frac{\mathrm{f}(x)}{\overline{\mathrm{F}}(t)}\right)^{\frac{\alpha}{\beta}} d x\right), \quad 0<\alpha<\beta, \beta \geq 1 .
$$

Obviously, when $t=0$, (8) reduces to (7).

An alternative way of expressing (8) is obtained in the following theorem: 
Theorem 1. For all $t>0$, we have

$$
\begin{aligned}
\mathrm{H}_{(\alpha, \beta)}^{\mathrm{w}}(X ; t)=\frac{\alpha}{\beta(\beta-\alpha)} \log \left[t^{\frac{\alpha}{\beta}} \exp \left(\frac{\beta(\beta-\alpha)}{\alpha} \mathrm{H}_{(\alpha, \beta)}(X ; t)\right)\right. \\
\left.+\frac{\alpha}{\beta} \int_{t}^{\infty} z^{\frac{\alpha}{\beta}-1}\left(\frac{\overrightarrow{\mathrm{F}}(z)}{\overline{\mathrm{F}}(t)}\right)^{\frac{\alpha}{\beta}} \exp \left(\frac{\beta(\beta-\alpha)}{\alpha} \mathrm{H}_{(\alpha, \beta)}(X ; z)\right) d z\right]
\end{aligned}
$$

Proof.

$$
\begin{aligned}
\int_{t}^{\infty}\left(x \frac{\mathrm{f}(x)}{\overline{\mathrm{F}}(t)}\right)^{\frac{\alpha}{\beta}} d x=\int_{t}^{\infty}\left(\int_{0}^{x} \frac{\alpha}{\beta} z^{\frac{\alpha}{\beta}-1} d z\right)\left(\frac{\mathrm{f}(x)}{\overline{\overline{\mathrm{F}}}(t)}\right)^{\frac{\alpha}{\beta}} d x \\
=\frac{\alpha}{\beta} \int_{t}^{\infty}\left[\int_{0}^{t} z^{\frac{\alpha}{\beta}-1} d z+\int_{t}^{x} z^{\frac{\alpha}{\beta}-1} d z\right]\left(\frac{\mathrm{f}(x)}{\overline{\overline{\mathrm{F}}}(t)}\right)^{\frac{\alpha}{\beta}} d x \\
=t^{\frac{\alpha}{\beta}} \int_{t}^{\infty}\left(\frac{\mathrm{f}(x)}{\overline{\mathrm{F}}(t)}\right)^{\frac{\alpha}{\beta}} d x+\frac{\alpha}{\beta} \int_{z=t}^{\infty}\left(\frac{1}{\overline{\mathrm{F}}^{\frac{\alpha}{\beta}}(t)} z^{\frac{\alpha}{\beta}-1}\left(\int_{x=z}^{\infty} \mathrm{f}^{\frac{\alpha}{\beta}}(x) d x\right) d z\right.
\end{aligned}
$$

From (4),

$$
\int_{t}^{\infty}\left(\frac{\mathrm{f}(x)}{\overline{\mathrm{F}}(t)}\right)^{\frac{\alpha}{\beta}} d x=\exp \left(\frac{\beta(\beta-\alpha)}{\alpha} \mathrm{H}_{(\alpha, \beta)}(X ; t)\right)
$$

and

$$
\int_{t}^{\infty} \mathrm{f}^{\frac{\alpha}{\beta}}(x) d x=\overline{\mathrm{F}}^{\frac{\alpha}{\beta}}(t) \exp \left(\frac{\beta(\beta-\alpha)}{\alpha} \mathrm{H}_{(\alpha, \beta)}(X ; t)\right) .
$$

Therefore, by using (10), (11), and (12) in (8), the desired result is obtained.

In Table 3, consider the general expressions of WGRE corresponding to some particular lifetime distributions. 


\section{BHAT \& BAIG}

Table 3. WGRE of some lifetime distributions

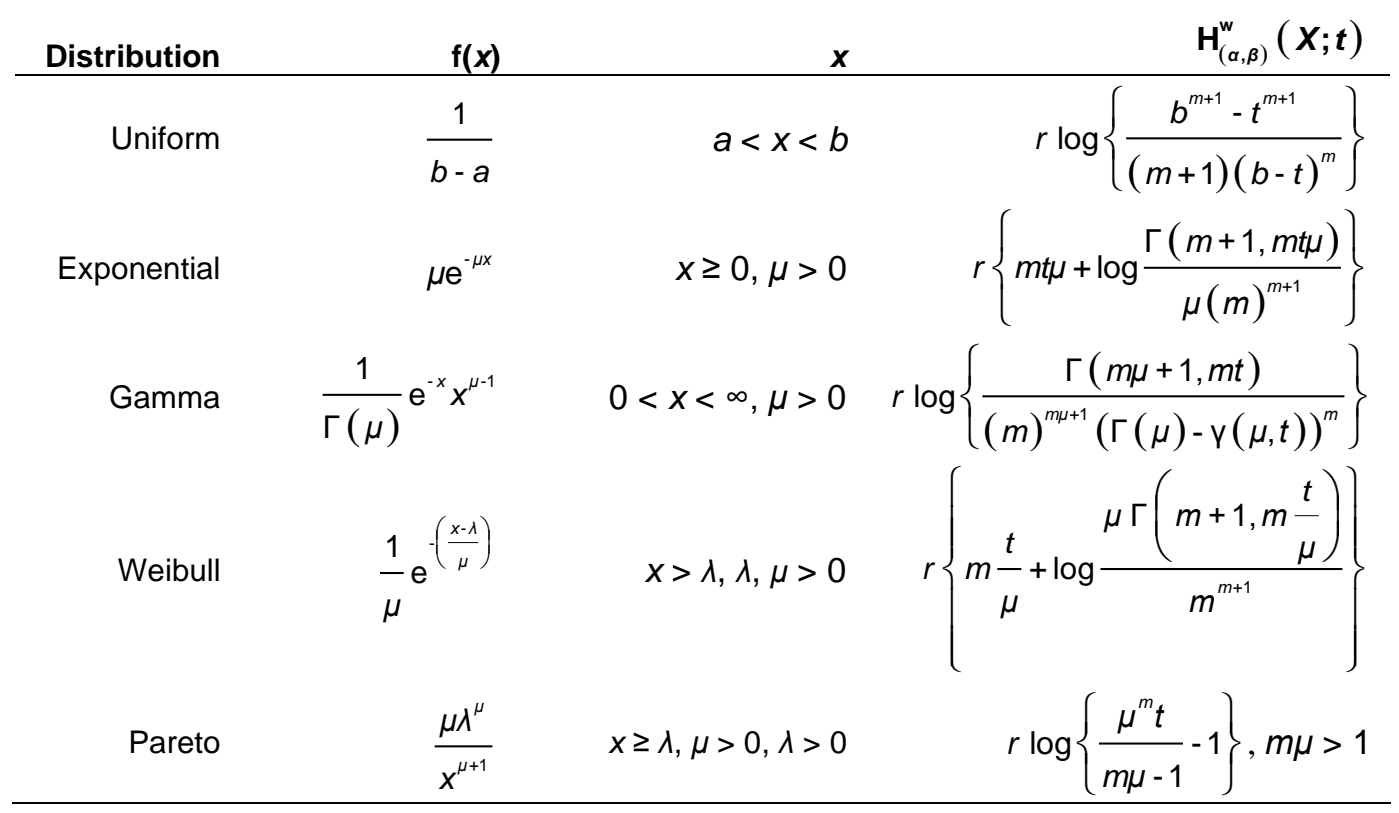

Note: $\quad\left\ulcorner(b, a y)=a^{b} \int_{y}^{\infty} \mathrm{e}^{-a u} u^{b-1} d u, a, b>0\right.$, and $\mathrm{\gamma}(b, a y)=a^{b} \int_{0}^{y} \mathrm{e}^{-a u} u^{b-1} d u, a, b>0$, are the upper and lower incomplete gamma functions; $m=\alpha / \beta, r=\alpha / \beta(\beta-\alpha)$

Corresponding to a well-known lifetime exponential distribution, consider the monotonic behavior of WGRE (8) with respect to parameters $\alpha$ and $\beta$.

For exponential distribution with $\mathrm{f}(x)=\mu \mathrm{e}^{-\mu x}, x \geq 0, \mu>0$,

$$
\mathrm{H}_{(\alpha, \beta)}^{\mathrm{w}}(X ; t)=r\left\{m t \mu+\log \frac{\Gamma(m+1, m t \mu)}{\mu(m)^{m+1}}\right\},
$$

where

$$
r=\frac{\alpha}{\beta(\beta-\alpha)} \quad \text { and } \quad m=\frac{\alpha}{\beta}
$$

The graphical behavior of (13) is exhibited in Figure 1. 


\section{TWO PARAMETRIC ENTROPY FOR LIFETIME DISTRIBUTIONS}
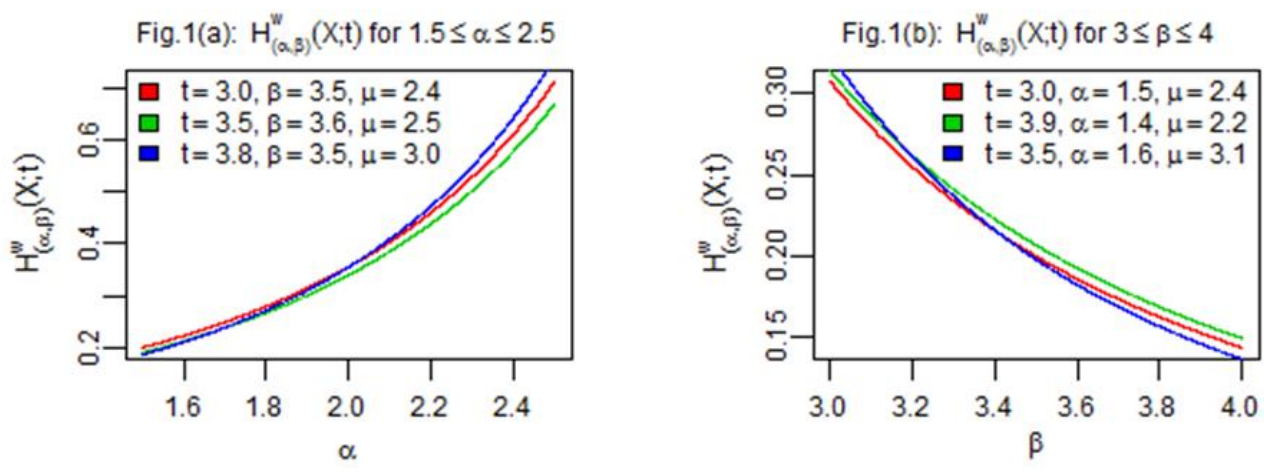

Figure 1. $\mathrm{H}_{(\alpha, \beta)}^{\mathrm{w}}(X ; t)$ plot of Exponential distribution with respect to $\alpha$ and $\beta$

It is clear from Figure 1 that $\mathrm{H}_{(\alpha, \beta)}^{\mathrm{w}}(X ; t)$ is monotone with respect to both $\alpha$ and $\beta$.

The following theorem proves that $\mathrm{H}_{(\alpha, \beta)}^{\mathrm{w}}(X ; t)$ characterizes $\overline{\mathrm{F}}(t)$ uniquely:

Theorem 2. Let $\mathrm{H}_{(\alpha, \beta)}^{\mathrm{w}}(X ; t)<\infty \forall 0<\alpha<\beta, \beta \geq 1$ and $\overline{\mathrm{F}}(t)$ be the WGRE and s.f. of a r.v. $X$. Assume that $\mathrm{H}_{(\alpha, \beta)}^{\mathrm{w}}(X ; t)$ is increasing in $t$, then $\mathrm{H}_{(\alpha, \beta)}^{\mathrm{w}}(X ; t)$ determines $\overline{\mathrm{F}}(t)$ uniquely.

Proof. From (8),

$$
\exp \left(\frac{\beta(\beta-\alpha)}{\alpha} \mathrm{H}_{(\alpha, \beta)}^{\mathrm{w}}(X ; t)\right)=\int_{t}^{\infty}\left(x \frac{\mathrm{f}(x)}{\overline{\mathrm{F}}(t)}\right)^{\frac{\alpha}{\beta}} d x
$$

Differentiating (14) w.r.t. $t$, obtain

$$
\frac{\partial}{\partial t} \exp \left(\frac{\beta(\beta-\alpha)}{\alpha} \mathrm{H}_{(\alpha, \beta)}^{\mathrm{w}}(X ; t)\right)=\frac{\alpha}{\beta} \lambda_{\mathrm{F}}(t) \int_{t}^{\infty}\left(x \frac{\mathrm{f}(x)}{\overline{\mathrm{F}}(t)}\right)^{\frac{\alpha}{\beta}} d x-\left(t \lambda_{\mathrm{F}}(t)\right)^{\frac{\alpha}{\beta}}
$$

where $\lambda_{\mathrm{F}}(t)=\mathrm{f}(t) / \overline{\mathrm{F}}(t)$ is the failure rate of $X$. Using (14), we can rewrite (15) as 


\section{BHAT \& BAIG}

$$
\begin{aligned}
\frac{\partial}{\partial t} \exp \left(\frac{\beta(\beta-\alpha)}{\alpha}\right. & \left.H_{(\alpha, \beta)}^{w}(X ; t)\right) \\
& =\frac{\alpha}{\beta} \lambda_{F}(t) \exp \left(\frac{\beta(\beta-\alpha)}{\alpha} H_{(\alpha, \beta)}^{w}(X ; t)\right)-\left(t \lambda_{F}(t)\right)^{\frac{\alpha}{\beta}}
\end{aligned}
$$

Rearranging (9),

$$
\begin{aligned}
\exp \left(\frac{\beta(\beta-\alpha)}{\alpha}\right. & \left.H_{(\alpha, \beta)}^{w}(X ; t)\right) \\
= & t^{\frac{\alpha}{\beta}} \exp \left(\frac{\beta(\beta-\alpha)}{\alpha} \mathrm{H}_{(\alpha, \beta)}(X ; t)\right) \\
& +\frac{\alpha}{\beta} \int_{t}^{\infty} z^{\frac{\alpha}{\beta}-1}\left(\frac{\overline{\mathrm{F}}(z)}{\overline{\mathrm{F}}(t)}\right)^{\frac{\alpha}{\beta}} \exp \left(\frac{\beta(\beta-\alpha)}{\alpha} \mathrm{H}_{(\alpha, \beta)}(X ; z)\right) d z
\end{aligned}
$$

Differentiating (17) w.r.t. $t$,

$$
\begin{aligned}
\frac{\partial}{\partial t} \exp ( & \left.\frac{\beta(\beta-\alpha)}{\alpha} H_{(\alpha, \beta)}^{w}(X ; t)\right) \\
= & t^{\frac{\alpha}{\beta}} \frac{\partial}{\partial t} \exp \left(\frac{\beta(\beta-\alpha)}{\alpha} \mathrm{H}_{(\alpha, \beta)}(X ; t)\right) \\
& +\left(\frac{\alpha}{\beta}\right)^{2} \lambda_{\mathrm{F}}(t) \int_{t}^{\infty} z^{\frac{\alpha}{\beta}-1}\left(\frac{\overline{\mathrm{F}}_{X}(z)}{\overline{\mathrm{F}}_{X}(t)}\right)^{\frac{\alpha}{\beta}} \exp \left(\frac{\beta(\beta-\alpha)}{\alpha} \mathrm{H}_{(\alpha, \beta)}(X ; z)\right) d z
\end{aligned}
$$

From (16) and (18),

$$
\begin{aligned}
& 0=\frac{\alpha}{\beta} \lambda_{\mathrm{F}}(t)\left[\frac { \alpha } { \beta } \int _ { t } ^ { \infty } z ^ { \frac { \alpha } { \beta } - 1 } \left(\frac{\left.\overline{\mathrm{F}}_{X}(z)\right)^{\frac{\alpha}{\beta}}}{\overline{\mathrm{F}}_{X}(t)} \exp \left(\frac{\beta(\beta-\alpha)}{\alpha} \mathrm{H}_{(\alpha, \beta)}(X ; z)\right) d z\right.\right. \\
& \left.-\exp \left(\frac{\beta(\beta-\alpha)}{\alpha} \mathrm{H}_{(\alpha, \beta)}^{\mathrm{w}}(X ; t)\right)\right]+t^{\frac{\alpha}{\beta}}\left(\lambda_{\mathrm{F}}(t)\right)^{\frac{\alpha}{\beta}}+t^{\frac{\alpha}{\beta}} \frac{\partial}{\partial t} \exp \left(\frac{\beta(\beta-\alpha)}{\alpha} \mathrm{H}_{(\alpha, \beta)}(X ; t)\right)
\end{aligned}
$$




\section{TWO PARAMETRIC ENTROPY FOR LIFETIME DISTRIBUTIONS}

Hence, for fixed $t>0, \lambda_{\mathrm{F}}(t)$ is a solution of $\psi\left(x_{t}\right)=0$, where

$$
\begin{aligned}
\psi\left(x_{t}\right) & =t^{\frac{\alpha}{\beta}} x_{t}^{\frac{\alpha}{\beta}}+\frac{\alpha}{\beta} x_{t}\left[\frac{\alpha}{\beta} \int_{t}^{\infty} z^{\frac{\alpha}{\beta}-1}\left(\frac{\overline{\mathrm{F}}_{X}(z)}{\overline{\mathrm{F}}_{X}(t)}\right)^{\frac{\alpha}{\beta}} \exp \left(\frac{\beta(\beta-\alpha)}{\alpha} \mathrm{H}_{(\alpha, \beta)}(X ; z)\right) d z\right. \\
& \left.-\exp \left(\frac{\beta(\beta-\alpha)}{\alpha} \mathrm{H}_{(\alpha, \beta)}^{\mathrm{w}}(X ; t)\right)\right]+t^{\frac{\alpha}{\beta}} \frac{\partial}{\partial t} \exp \left(\frac{\beta(\beta-\alpha)}{\alpha} \mathrm{H}_{(\alpha, \beta)}(X ; t)\right)
\end{aligned}
$$

Differentiating both sides w.r.t. $x_{t}$,

$$
\begin{aligned}
\psi^{\prime}\left(x_{t}\right)= & \frac{\alpha}{\beta} t^{\frac{\alpha}{\beta}} x^{\frac{\alpha}{\beta}-1}+\frac{\alpha}{\beta}\left[\frac{\alpha}{\beta} \int_{t}^{\infty} z^{\frac{\alpha}{\beta}-1}\left(\frac{\overline{\mathrm{F}}_{X}(z)}{\overline{\mathrm{F}}_{X}(t)}\right)^{\frac{\alpha}{\beta}} \exp \left(\frac{\beta(\beta-\alpha)}{\alpha} \mathrm{H}_{(\alpha, \beta)}(X ; z)\right) d z\right. \\
& \left.-\exp \left(\frac{\beta(\beta-\alpha)}{\alpha} \mathrm{H}_{(\alpha, \beta)}^{\mathrm{w}}(X ; t)\right)\right]
\end{aligned}
$$

Now, $\psi^{\prime}\left(x_{t}\right)=0$ gives

$$
\begin{aligned}
& x_{t}=t^{-\frac{\alpha}{\beta}}\left[\exp \left(\frac{\beta(\beta-\alpha)}{\alpha} \mathrm{H}_{(\alpha, \beta)}^{\mathrm{w}}(X ; t)\right)\right. \\
& \left.\quad-\frac{\alpha}{\beta} \int_{t}^{\infty} z^{\frac{\alpha}{\beta}-1}\left(\frac{\overline{\mathrm{F}}_{X}(z)}{\overline{\mathrm{F}}_{X}(t)}\right)^{\frac{\alpha}{\beta}} \exp \left(\frac{\beta(\beta-\alpha)}{\alpha} \mathrm{H}_{(\alpha, \beta)}(X ; z)\right) d z\right]^{\frac{\beta}{\alpha-\beta}} \\
& =x_{0} \text { (say) }
\end{aligned}
$$

Also,

$$
\psi^{\prime \prime}\left(x_{t}\right)=\frac{\alpha}{\beta}\left(\frac{\alpha}{\beta}-1\right) t^{\frac{\alpha}{\beta}} x^{\frac{\alpha}{\beta}-2} .
$$

For $0<\alpha<\beta, \beta \geq 1, \psi^{\prime \prime}\left(x_{t}\right)<0$. Thus $\psi\left(x_{t}\right)$ attains maximum at $\left(x_{0}\right)$. Also, $\psi(0)>0$ and $\psi(\infty)=-\infty$. Further, $\psi\left(x_{t}\right)$ increases for $0<x_{\mathrm{t}}<x_{0}$ and decreases for $x_{t}>x_{0}$. 


\section{BHAT \& BAIG}

The unique solution to $\psi\left(x_{t}\right)=0$ is given by $x_{t}=\lambda_{\mathrm{F}}(t)$. Thus, $\mathrm{H}_{(\alpha, \beta)}^{\mathrm{w}}(X ; t)$ uniquely determines $\lambda_{\mathrm{F}}(t)$, which in turns determines $\overline{\mathrm{F}}(t)$.

\section{Properties and Inequalities of WGRE}

Definition 2. A r.v. $X$ is said to be smaller than the other r.v. $Y$ in WGRE (denoted by $X \stackrel{\text { WGRE }}{\leq} Y$ if

$$
\mathrm{H}_{(\alpha, \beta)}^{\mathrm{w}}(X ; t) \leq \mathrm{H}_{(\alpha, \beta)}^{\mathrm{w}}(Y ; t), \forall t>0 .
$$

Definition 3. A survival function $\overline{\mathrm{F}}$ is said to have increasing (decreasing) WGRE of order $\alpha$ and type $\beta$ denoted by IWGRE (DWGRE) if $\mathrm{H}_{(\alpha, \beta)}^{\mathrm{w}}(X ; t)$ is increasing (decreasing) in $t, t>0$.

Theorem 3. Let $\overline{\mathrm{F}}$ be an IWGRE (DWGRE) and $\beta>\alpha$, then

$$
\lambda_{\mathrm{F}}(t) \leq(\geq)\left[\frac{\alpha \exp \left(\frac{\beta(\beta-\alpha)}{\alpha} \mathrm{H}_{(\alpha, \beta)}^{\mathrm{w}}(X ; t)\right)}{\beta t^{\frac{\alpha}{\beta}}}\right]^{\frac{\beta}{\alpha-\beta}}
$$

Proof. From (8),

$$
\frac{\beta(\beta-\alpha)}{\alpha} \frac{\partial}{\partial t} \mathrm{H}_{(\alpha, \beta)}^{\mathrm{w}}(X ; t)=\lambda_{\mathrm{F}}(t)\left[\frac{\alpha}{\beta}-t^{\frac{\alpha}{\beta}} \lambda_{\mathrm{F}}(t)^{\frac{\alpha}{\beta}-1} \exp \left(-\frac{\beta(\beta-\alpha)}{\alpha} \mathrm{H}_{(\alpha, \beta)}^{\mathrm{w}}(X ; t)\right)\right]
$$

Because $\overline{\mathrm{F}}$ is IWGRE (DWGRE) and $\beta>\alpha$,

$$
\lambda_{\mathrm{F}}(t)\left[\frac{\alpha}{\beta}-t^{\frac{\alpha}{\beta}} \lambda_{\mathrm{F}}^{\frac{\alpha}{\beta}-1}(t) \exp \left(-\frac{\beta(\beta-\alpha)}{\alpha} \mathrm{H}_{(\alpha, \beta)}^{\mathrm{w}}(X ; t)\right)\right] \geq(\leq) 0
$$

which leads to 


$$
\lambda_{\mathrm{F}}(t) \leq(\geq)\left[\frac{\alpha \exp \left(\frac{\beta(\beta-\alpha)}{\alpha} \mathrm{H}_{(\alpha, \beta)}^{\mathrm{w}}(X ; t)\right)}{\beta t^{\frac{\alpha}{\beta}}}\right]^{\frac{\beta}{\alpha-\beta}} .
$$

Example 2. Let $X$ be a r.v. with $\mathrm{f}(x)=\mu \mathrm{e}^{-\theta x}, x>0, \theta>0$, then from Table 3,

$$
\frac{\partial}{\partial t} \mathrm{H}_{(\alpha, \beta)}^{\mathrm{w}}(X ; t)=\frac{\alpha}{\beta(\beta-\alpha)}\left[\frac{\alpha}{\beta} \theta+\frac{\Gamma^{\prime}\left(\frac{\alpha}{\beta}+1, \frac{\alpha}{\beta} \theta t\right)}{\Gamma\left(\frac{\alpha}{\beta}+1, \frac{\alpha}{\beta} \theta t\right)}\right] .
$$

Therefore, if $\beta>\alpha, \overline{\mathrm{F}}$ is IWGRE.

Theorem 4. If the r.v. $X$ has

(I) IWGRE, then $\mathrm{H}_{(\alpha, \beta)}^{\mathrm{w}}(X ; t)$ obtains a lower bound as follows:

$$
\mathrm{H}_{(\alpha, \beta)}^{\mathrm{w}}(X ; t) \geq \frac{\alpha}{\beta(\beta-\alpha)} \log \left(\frac{\beta t^{\frac{\alpha}{\beta}}}{\alpha}\left(\frac{1+\frac{\partial}{\partial t} \mathrm{~m}_{\mathrm{F}}(t)}{\mathrm{m}_{\mathrm{F}}(t)}\right)^{\frac{\alpha}{\beta}-1}\right) .
$$

(II) DWBGRE, then $\mathrm{H}_{(\alpha, \beta)}^{\mathrm{w}}(X ; t)$ obtains an upper bound as follows:

$$
\mathrm{H}_{(\alpha, \beta)}^{\mathrm{w}}(X ; t) \leq \frac{\alpha}{\beta(\beta-\alpha)} \log \left(\frac{\beta t^{\frac{\alpha}{\beta}}}{\alpha}\left(\frac{1+\frac{\partial}{\partial t} \mathrm{~m}_{\mathrm{F}}(t)}{\mathrm{m}_{\mathrm{F}}(t)}\right)^{\frac{\alpha}{\beta}-1}\right) .
$$

Proof. From (8), 


\section{BHAT \& BAIG}

$$
\frac{\beta(\beta-\alpha)}{\alpha} \frac{\partial}{\partial t} \mathrm{H}_{(\alpha, \beta)}^{\mathrm{w}}(X ; t)=\left[\frac{\alpha}{\beta} \lambda_{\mathrm{F}}(t)-\left(t \lambda_{\mathrm{F}}(t)\right)^{\frac{\alpha}{\beta}} \exp \left(-\frac{\beta(\beta-\alpha)}{\alpha} \mathrm{H}_{(\alpha, \beta)}^{\mathrm{w}}(X ; t)\right)\right]
$$

Substituting

$$
\lambda_{\mathrm{F}}(t)=\frac{1+\frac{\partial}{\partial t} \mathrm{~m}_{\mathrm{F}}(t)}{\mathrm{m}_{\mathrm{F}}(t)}
$$

where $\mathrm{m}_{\mathrm{F}}(t)$ is the mean residual life function of $X$,

$$
\begin{array}{r}
\frac{\partial}{\partial t} \mathrm{H}_{(\alpha, \beta)}^{\mathrm{w}}(X ; t)=\left(\frac{\alpha}{\beta(\beta-\alpha)}\right)\left[\frac{\alpha}{\beta}\left(\frac{1+\frac{\partial}{\partial t} \mathrm{~m}_{\mathrm{F}}(t)}{\mathrm{m}_{\mathrm{F}}(t)}\right)\right. \\
\left.-t^{\frac{\alpha}{\beta}}\left(\frac{1+\frac{\partial}{\partial t} \mathrm{~m}_{\mathrm{F}}(t)}{\mathrm{m}_{\mathrm{F}}(t)}\right)^{\frac{\alpha}{\beta}} \exp \left(-\frac{\beta(\beta-\alpha)}{\alpha} \mathrm{H}_{(\alpha, \beta)}^{\mathrm{w}}(X ; t)\right)\right]
\end{array}
$$

Because $\mathrm{H}_{(\alpha, \beta)}^{\mathrm{w}}(X ; t)$ is increasing w.r.t. $t$,

$$
\mathrm{H}_{(\alpha, \beta)}^{\mathrm{w}}(X ; t) \geq \frac{\alpha}{\beta(\beta-\alpha)} \log \left(\frac{\beta t^{\frac{\alpha}{\beta}}}{\alpha}\left(\frac{1+\frac{\partial}{\partial t} \mathrm{~m}_{\mathrm{F}}(t)}{\mathrm{m}_{\mathrm{F}}(t)}\right)^{\frac{\alpha}{\beta}-1}\right)
$$

The proof of (II) is similar.

Theorem 5. Let $X$ be the lifetime of a system with p.d.f. $\mathrm{f}(x)$ and s.f. $\overline{\mathrm{F}}(x), t>0$, then $\mathrm{H}_{(\alpha, \beta)}^{\mathrm{w}}(X ; t)$ attains a lower bound of as follows: 


$$
\mathrm{H}_{(\alpha, \beta)}^{\mathrm{w}}(X ; t) \geq \frac{\alpha^{2}}{\beta^{2}(\beta-\alpha)} \int_{t}^{\infty} \frac{\mathrm{f}(x)}{\overline{\mathrm{F}}(t)} \log x d x+\frac{\alpha}{\beta^{2}} \mathrm{H}(X ; t) .
$$

Proof. From the log-sum inequality,

$$
\begin{aligned}
\int_{t}^{\infty} \mathrm{f}(x) \log \frac{\mathrm{f}(x)}{\left(x \frac{\mathrm{f}(x)}{\overline{\mathrm{F}}(t)}\right)^{\frac{\alpha}{\beta}}} d x & \geq\left(\int_{t}^{\infty} \mathrm{f}(x) d x\right) \log \left(\frac{\int_{t}^{\infty} \mathrm{f}(x) d x}{\left.\int_{t}^{\infty}\left(x \frac{\mathrm{f}(x)}{\overline{\mathrm{F}}(t)}\right)^{\frac{\alpha}{\beta}} d x\right)}\right. \\
& =\overline{\mathrm{F}}(t)\left(\log \overline{\mathrm{F}}(t)-\frac{\beta(\beta-\alpha)}{\alpha} \mathrm{H}_{(\alpha, \beta)}^{\mathrm{w}}(X ; t)\right)
\end{aligned}
$$

where (19) is obtained from (8).

The L.H.S. of (19) leads to

$$
\left(1-\frac{\alpha}{\beta}\right) \int_{t}^{\infty} \mathrm{f}(x) \log \mathrm{f}(x) d x-\frac{\alpha}{\beta} \int_{t}^{\infty} \mathrm{f}(x) \log x d x+\frac{\alpha}{\beta} \overline{\mathrm{F}}(t) \log \overline{\mathrm{F}}(t) .
$$

Substituting (20) in (19) leads to the desired result.

Consider a lemma which will be very useful in proving some of the theorems in the section.

Lemma 1. Let $X$ be a r.v. and define $Z=a X$, where $a>0$ is a constant, then the following equality holds:

$$
\mathrm{H}_{(\alpha, \beta)}^{\mathrm{w}}(Z ; t)=\frac{\alpha}{\beta(\beta-\alpha)} \log a+\mathrm{H}_{(\alpha, \beta)}^{\mathrm{w}}\left(X ; \frac{t}{a}\right) .
$$

\section{Proof.}

$$
\mathrm{H}_{(\alpha, \beta)}^{\mathrm{w}}(Z ; t)=\frac{\alpha}{\beta(\beta-\alpha)} \log \int_{t}^{\infty}\left(\frac{z \mathrm{f}\left(\frac{z}{a}\right)}{a \operatorname{Pr}(Z>t)}\right)^{\frac{\alpha}{\beta}} d z .
$$




\section{BHAT \& BAIG}

Setting $Z=a X$, a strictly increasing function of $X$,

$$
\mathrm{H}_{(\alpha, \beta)}^{\mathrm{w}}(Z ; t)=\frac{\alpha}{\beta(\beta-\alpha)} \log \left[a \int_{\frac{t}{a}}^{\infty}\left(x \frac{\mathrm{f}(x)}{\overline{\mathrm{F}}\left(\frac{t}{a}\right)}\right)^{\frac{\alpha}{\beta}} d x\right]
$$

By using (8), the desired result is obtained.

Theorem 6. Let $X$ and $Y$ be two r.v.s. Define $Z_{1}=a_{1} X$ and $Z_{2}=a_{2} Y, a_{1}, a_{2}>0$. Let $X \stackrel{\text { WGRE }}{\leq} Y$ and $a_{1} \leq a_{2}$; then $Z_{1} \stackrel{\text { WGRE }}{\leq} Z_{2}$.

Proof. Suppose $\mathrm{H}_{(\alpha, \beta)}^{\mathrm{w}}(X ; t)$ is decreasing in $t$. Now, $X \stackrel{\text { WGRE }}{\leq} Y$ implies

$$
\mathrm{H}_{(\alpha, \beta)}^{\mathrm{w}}\left(X ; \frac{t}{a_{2}}\right) \leq \mathrm{H}_{(\alpha, \beta)}^{\mathrm{w}}\left(Y ; \frac{t}{a_{2}}\right) .
$$

Because $t / a_{1} \geq t / a_{2}$

$$
\mathrm{H}_{(\alpha, \beta)}^{\mathrm{w}}\left(X ; \frac{t}{a_{1}}\right) \leq \mathrm{H}_{(\alpha, \beta)}^{\mathrm{w}}\left(X ; \frac{t}{a_{2}}\right)
$$

Combining (21) and (22),

$$
\mathrm{H}_{(\alpha, \beta)}^{\mathrm{w}}\left(X ; \frac{t}{a_{1}}\right) \leq \mathrm{H}_{(\alpha, \beta)}^{\mathrm{w}}\left(Y ; \frac{t}{a_{2}}\right) .
$$

Using Lemma 1 in (23), $Z_{1} \stackrel{\text { WGRE }}{\leq} Z_{2}$.

Theorem 7. For the r.v. $X$ having support $(0, m]$, p.d.f. $\mathrm{f}(x)$ and s.f. $\overline{\mathrm{F}}(t), t>0$, then the following upper bound of $\mathrm{H}_{(\alpha, \beta)}^{\mathrm{w}}(X ; t)$ holds: 
TWO PARAMETRIC ENTROPY FOR LIFETIME DISTRIBUTIONS

$$
\mathrm{H}_{(\alpha, \beta)}^{\mathrm{w}}(X ; t) \leq \frac{\alpha}{\beta(\beta-\alpha)}\left[\frac{\int_{t}^{m}\left(x \frac{\mathrm{f}(x)}{\overline{\mathrm{F}}(t)}\right)^{\frac{\alpha}{\beta}} \log \left(x \frac{f(x)}{\bar{F}(t)}\right)^{\frac{\alpha}{\beta}} d x}{\int_{t}^{b}\left(x \frac{\mathrm{f}(x)}{\overline{\overline{\mathrm{F}}}(t)}\right)^{\frac{\alpha}{\beta}} d x}+\log (m-t)\right]
$$

Proof. From log-sum inequality and (8),

$$
\begin{aligned}
& \int_{t}^{m}\left(x \frac{\mathrm{f}(x)}{\overline{\mathrm{F}}(t)}\right)^{\frac{\alpha}{\beta}} \log \left(x \frac{f(x)}{\bar{F}(t)}\right)^{\frac{\alpha}{\beta}} d x \geq \int_{t}^{m}\left(x \frac{\mathrm{f}(x)}{\overline{\mathrm{F}}(t)}\right)^{\frac{\alpha}{\beta}} d x \log \left(\frac{\int_{t}^{m}(x \mathrm{f}(x))^{\frac{\alpha}{\beta}} d x}{\int_{t}^{m}(\overline{\mathrm{F}}(t))^{\frac{\alpha}{\beta}} d x}\right) \\
& =\int_{t}^{m}\left(x \frac{\mathrm{f}(x)}{\overline{\mathrm{F}}(t)}\right)^{\frac{\alpha}{\beta}} d x\left[\frac{\beta(\beta-\alpha)}{\alpha} \mathrm{H}_{(\alpha, \beta)}^{\mathrm{w}}(X ; t)-\log (m-t)\right]
\end{aligned}
$$

After simplification, the proof is obvious.

Proposition 2. For the r.v. $X$ having WGRE $\mathrm{H}_{(\alpha, \beta)}^{\mathrm{w}}(X ; t)$,

$$
\mathrm{H}_{(\alpha, \beta)}^{\mathrm{w}}(X ; t) \leq \frac{\alpha}{\beta(\beta-\alpha)}\left[\int_{t}^{\infty}\left(x \frac{\mathrm{f}(x)}{\overline{\mathrm{F}}(t)}\right)^{\frac{\beta}{\alpha}} d x-1\right] .
$$

Proof. Since, for every positive $x \in \mathbb{R}, \log x \leq x-1$,

$$
\begin{aligned}
\mathrm{H}_{(\alpha, \beta)}^{\mathrm{w}}(X ; t) & =\frac{\alpha}{\beta(\beta-\alpha)} \log \int_{t}^{\infty}\left(x \frac{\mathrm{f}(x)}{\overline{\mathrm{F}}(t)}\right)^{\frac{\alpha}{\beta}} d x \\
& \leq \frac{\alpha}{\beta(\beta-\alpha)}\left[\int_{t}^{\infty}\left(x \frac{\mathrm{f}(x)}{\overline{\mathrm{F}}(t)}\right)^{\frac{\alpha}{\beta}} d x-1\right]
\end{aligned}
$$




\section{BHAT \& BAIG}

Theorem 8. Let $X$ be IWGRE (DWGRE) and define $Z=a X$, where $a>0$ is a constant. Then $Z$ is IWGRE (DWGRE).

Proof. Because $X$ is IWGRE (DWGRE),

$$
\frac{\partial}{\partial t} \mathrm{H}_{(\alpha, \beta)}^{\mathrm{w}}(X ; t) \geq(\leq) 0
$$

By applying Lemma 1, it follows that $Z$ is IWGRE (DWGRE).

Finally, for Pareto distribution, consider the general expressions of all the uncertainty measures that are mentioned in this article.

Table 4. Expressions of different entropies with respect to Pareto distribution

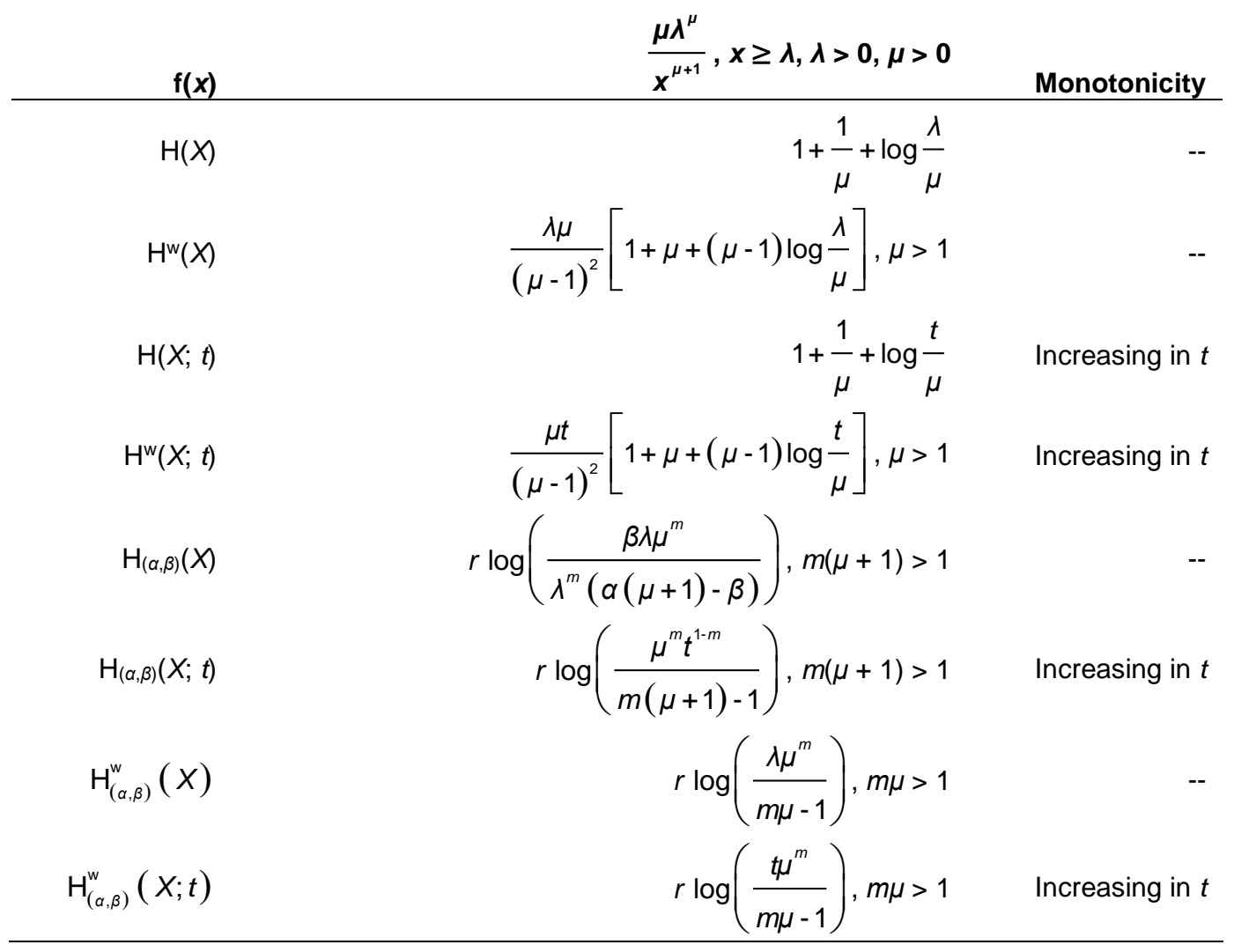

Note: $m=\alpha / \beta, r=\alpha / \beta(\beta-\alpha)$ 


\section{TWO PARAMETRIC ENTROPY FOR LIFETIME DISTRIBUTIONS}

\section{Conclusion}

A weighted generalized entropy of order $\alpha$ and type $\beta$ and its dynamic (residual) version were developed. The expressions of these measures were considered for some particular lifetime distributions. The proposed dynamic measure characterizes the distribution function uniquely. The various significant properties and inequalities of the dynamic measure were explored.

\section{References}

Belis, M., \& Guiasu, S. (1968). A quantitative-qualitative measure of information in cybernetic systems. IEEE Transactions on Information Theory, 14(4), 593-594. doi: 10.1109/TIT.1968.1054185

Di Crescenzo, A., \& Longobardi, M. (2006). On weighted residual and past entropies. Scientiae Mathematicae Japonicae, 64(2), 255-266.

Ebrahimi, N. (1996). How to measure uncertainty in the residual lifetime distribution. Sankhyā: The Indian Journal of Statistics, Series A, 58(1), 48-56.

Kayal, S. (2018). On weighted generalized cumulative residual entropy of order $n$. Methodology and Computing in Applied Probability, 20, 487-503. doi: 10.1007/s11009-017-9569-0

Khammar, A. H., \& Jahanshahi, S. M. A. (2018). On weighted cumulative residual Tsallis entropy and its dynamic version. Physica A: Statistical Mechanics and its Applications, 491, 678-692. doi: 10.1016/j.physa.2017.09.079

Mirali, M., \& Baratpour, S. (2017). Dynamic version of weighted cumulative residual entropy. Communications in Statistics - Theory and Methods, 46(22), 11047-11059. doi: 10.1080/03610926.2016.1257711

Misagh, F., Panahi, Y., Yari, G. H., \& Shahi, R. (2011, September 14-17). Weighted cumulative entropy and its estimation [Conference presentation]. 2011 IEEE International Conference on Quality and Reliability, Bangkok, Thailand. doi: 10.1109/ICQR.2011.6031765

Misagh, F., \& Yari, G. H. (2011). On weighted interval entropy. Statistics \& Probability Letters, 81(2), 188-194. doi: 10.1016/j.spl.2010.11.006

Nair, R. S., Abdul-Sathar, E. I., \& Rajesh, G. (2017). A study on dynamic weighted failure entropy of order $\alpha$. American Journal of Mathematical and Management Sciences, 36(2), 137-149. doi: 10.1080/01966324.2017.1298063 


\section{BHAT \& BAIG}

Nourbakhsh, M., \& Yari, G. (2016). Weighted Renyi's entropy for lifetime distributions. Communications in Statistics - Theory and Methods, 46(14), 70857098. doi: 10.1080/03610926.2016.1148729

Shannon, C. E. (1948). A mathematical theory of communications. Bell System Technical Journal, 27, 379-423.

Shiwei, Y., \& Ting-Zhu, H. (2017). Exponential weighted entropy and exponential weighted mutual information. Neurocomputing, 249, 86-94. doi: 10.1016/j.neucom.2017.03.075

Yasaei Sekeh, S., Mohtashami Borzadaran, G. R., \& Rezaei Roknabadi, A. H. (2012). Some results based on weighted dynamic entropies. Rendiconti del Seminario Matematico, 70(4), 369-382. Retrieved from

http://www.seminariomatematico.polito.it/rendiconti/70-4/369.pdf 